\title{
Viability of using Convolutional Neural Networks for Real-Time Fescue and Sheep Sorrel Detection in Wild Blueberry Fields
}

\author{
Patrick J. Hennessy ${ }^{1 *}$, Travis J. Esau ${ }^{1}$, Qamar U. Zaman ${ }^{1}$, Kenneth W. Corscadden ${ }^{2}$, Arnold W. Schumann ${ }^{3}$, Aitazaz \\ A. Farooque \\ ${ }^{1}$ Department of Engineering, Dalhousie University, Truro, Canada \\ ${ }^{2}$ Centre for Technology, Environment, \& Design, Lethbridge College, Lethbridge, Canada \\ ${ }^{3}$ Citrus Research and Education Center, University of Florida, Lake Alfred, United States \\ ${ }^{4}$ School of Sustainable Design Engineering, University of Prince Edward Island, Charlottetown, Canada \\ *Patrick.Hennessy@dal.ca
}

\begin{abstract}
Wild blueberry (Vaccinium angustifolium Ait.) production is hindered by weeds such as fescue (Festuca filiformis Pourr.) and sheep sorrel (Rumex acetosella L.). Commercial sprayers provide a uniform application of herbicide regardless of the incidence of weed coverage. Traditional methods of spot-applying herbicide to target weed locations relied on colour co-occurrence matrices that were limited by long processing times in addition to green colour segmentation lacking the ability to discriminate between weeds and crop canopy of similar colour. Deep learning convolutional neural networks (CNNs) are a modern processing technique which often uses powerful graphics processing units (GPUs) to classify images or detect objects within images. This novel research study featured two object-detection CNNs, YOLOv3 and YOLOv3-Tiny, trained to detect fescue using images of wild blueberry fields captured during application timing intervals in the 2019 growing season. A custom-built desktop computer using the Ubuntu 16.04 operating system and an NVIDIA GeForce RTX 2080 Ti GPU was used to test the CNNs using 1280x720 resolution images. YOLOv3 classified fescue images with an F1-score of 0.95 and YOLOv3-Tiny classified fescue images with an F1-score of 0.97. YOLOv3 and YOLOv3-Tiny classified sheep sorrel images with F1scores of 0.93 and 0.89 respectively. A laptop running Windows 10 Pro with an NVIDIA Quadro RTX 5000 GPU was used to process video streams from four USB cameras simultaneously. YOLOv3 processed each video stream at an average framerate of 4.7 FPS using 9.2 GB of vRAM, while YOLOv3-Tiny processed each video stream at an average framerate of 20.5 FPS using $3.2 \mathrm{~GB}$ of vRAM. Initial results suggest that YOLOv3-Tiny can be deployed for use with a machine vision system to detect fescue and sheep sorrel in realtime for spot application of herbicide in wild blueberry fields. Using CNNs to selectively spray herbicide will appreciably reduce the volume of herbicides needed to manage wild blueberry fields, resulting in cost-savings for producers.
\end{abstract}

Keywords-Deep learning; artificial intelligence; machine vision; precision agriculture; weed detection

Funding for this research was generously provided by the Natural Sciences and Engineering Research Council (NSERC) and Doug Bragg Enterprises, Ltd.

\section{INTRODUCTION}

Wild blueberries (Vaccinium angustifolium Ait.) are a perennial crop native to northeastern North America with a total production of over 108 million $\mathrm{kg}$ in 2019 [1]. The plants grow in a two-year cycle in which the flower buds begin to grow from August to October in the first (sprout) year and lay dormant during the winter. The plants continue growing in the second (crop) year with fruit being harvested in August and September. The bare branches are pruned by flail mowing or burning after harvesting [2].

Weeds are a major yield limiting factor in wild blueberry production [3]-[5], and are typically managed with application of liquid herbicides [3], [6]. In 2019, sheep sorrel (Rumex acetosella L.) and fescue (Festuca filiformis Pourr.) were respectively the first and fourth most common weeds in Nova Scotia wild blueberry fields [7]. Fescue can be managed using spring sprout year applications of pronamide or glufosinate [8]. Hexazinone and pronamide applications have given mixed results for managing sheep sorrel [3], [9], [10], but tests using spring applications of sulfentrazone have shown promising results [7]. Sheep sorrel and fescue had field uniformities of $63 \%$ and $25 \%$ respectively in 2019 [7], suggesting that uniform application methods waste herbicide. An opportunity for improved application efficiency is possible through targeting and spraying only areas of the fields with weed cover.

Smart sprayers use sensors to intelligently select which areas of a field to apply agrochemicals, reducing the volume of agrochemical needed for field management. Smart sprayers in wild blueberry have previously relied on imaging data to detect foliage [11]-[15] or ultrasonic sensors to detect plant height [16] for spraying herbicides. The smart sprayer relying on ultrasonic sensors could successfully detect weeds taller than the wild blueberry plant canopy but could not detect weeds at the same height or shorter than the canopy [16]. An imaging system which used green colour segmentation could successfully isolate target weeds from blueberry branches and bare ground and resulted in herbicide savings of up to $78.5 \%$ 
[13]. However, this system could not discriminate different weed species of the same colour. Another imaging system relying on colour co-occurrence matrices successfully identified goldenrod (Solidago Spp.) in wild blueberry fields, but had to be purpose-built for goldenrod and had slow processing speeds in certain situations [14].

Deep learning convolutional neural networks (CNNs) are an advanced form of image processing which can quickly and accurately classify images or objects within an image [17]. CNNs are trained to detect targets using backpropagation, which involves showing a computer many labelled images of the desired target [18]. Training through backpropagation automatically determines the most important visual features of the targets, limiting the involvement from the user. CNNs are typically trained and processed using graphics processing units (GPUs) because they have many more processing cores than a central processing unit (CPU), which allows the calculations to be performed faster through parallelization [17], [19]. Image processing using CNNs has been used in various aspects of agriculture since 2015 [20]. Innovative uses of this technology in agriculture have included livestock monitoring [21]-[23], plant disease detection [24]-[26], wild blueberry ripeness detection [27], and weed detection for strawberries [28], Florida vegetables [29], turfgrasses [30], [31], and ryegrass [32]. Reference [33] was the first to use CNNs for detecting weeds in wild blueberry fields. The authors of [33] discussed that although not all instances of fescue and sheep sorrel were detected, only one detection per image was needed to trigger a spray event. The authors also noted that multiple cameras, and therefore multiple $\mathrm{CNN}$ instances, would be required to detect weeds underneath each sprayer nozzle.

This study evaluated two CNNs, YOLOv3 and YOLOv3Tiny [34], to determine if they could accurately detect at least one instance of fescue or sheep sorrel in images. These CNNs were previously trained by [33] to detect fescue and sheep sorrel in images of wild blueberry fields in Nova Scotia,

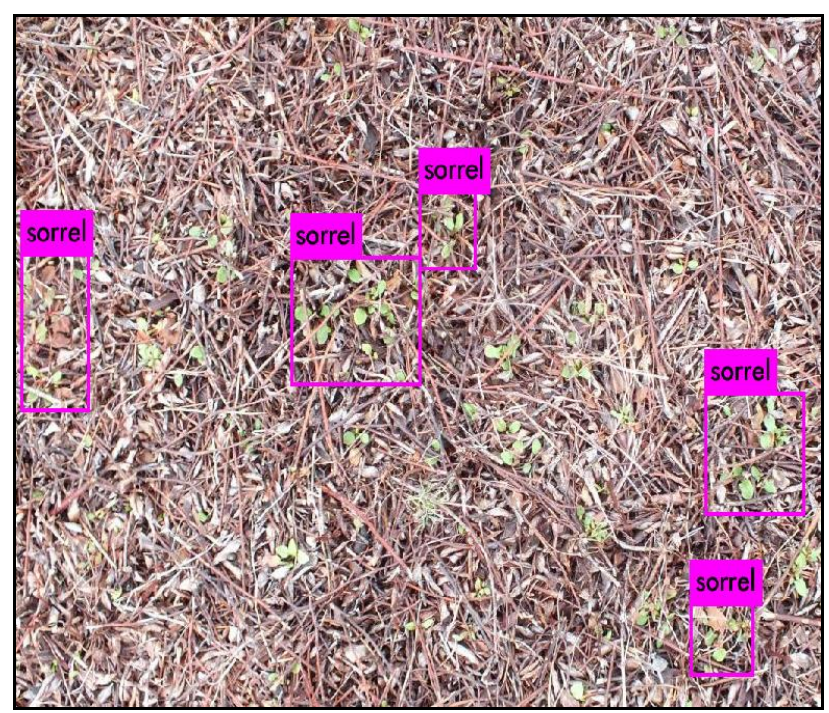

Figure 1. Sheep sorrel detections using YOLOv3-Tiny at 1280x736 resolution for an image from the Cattle Market field $\left(45.3674^{\circ} \mathrm{N}\right.$, $63.2124^{\circ} \mathrm{W}$ ) captured on April 24, 2019 [33]. The round, green leaves are sheep sorrel plants growing around wild blueberry branches leftover from flail mowing the previous autumn.
Canada. Furthermore, this study examined the processing speed and memory requirements of a mobile GPU when processing multiple instances of the CNNs to determine if it would be viable for controlling spray applications. Using CNNs to control spray applications could lead to greater herbicide application efficiency, resulting in major cost savings for wild blueberry producers.

\section{MATERIALS AND METHODS}

The ability of the CNNs to detect fescue and sheep sorrel was measured using separate datasets each containing 800 images. The dataset for testing fescue detection contained 402 images with one or more instances of fescue and 398 images without any instances of fescue. The dataset for sheep sorrel detection contained 394 images with at least one instance of sheep sorrel, and 406 images without any sheep sorrel. The images were captured from April 24 to May 17, 2019 in northern and central Nova Scotia from sprout year fields. The images were originally captured using eight digital cameras with resolutions from $4000 \times 3000$ to $6000 \times 4000$ pixels, then were scaled and cropped to $1280 \times 720$ pixels using IrfanView ${ }^{1}$ for processing. The locations of target weeds in the images were labelled with software developed by [27].

A custom-built desktop computer containing an NVIDIA

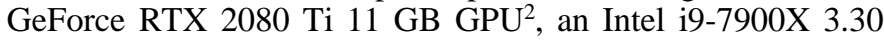
$\mathrm{GHz} \mathrm{CPU}^{3}$, and $32 \mathrm{~GB}$ of random-access memory (RAM) running the Ubuntu 16.04 operating system ${ }^{4}$ was used to validate the CNNs. The Darknet framework [35] was installed to process the CNNs using the weight files trained by [33]. YOLOv3 and YOLOv3-Tiny were tested at three different network resolutions $(1280 \times 736,1024 \times 576$, and $960 \times 544)$ to determine the effect that resolution had on detection accuracy. Reference [33] previously determined the precision, recall, and F1-score metrics [36] for the networks at detecting all instances of target weeds in images. These metrics are functions of true positive $(t p)$, false positive $(f p)$, and false negative $(f n)$ detections of targets. Precision is ratio of true positives to all detections:

$$
P r=\frac{t p}{t p+f p}
$$

Recall is the ratio of true positives to all relevant targets:

$$
R e=\frac{t p}{t p+f n}
$$

F1-score is the harmonic mean of precision and recall:

$$
F I=2 \cdot \frac{P r \cdot R e}{P r+R e}
$$

Considering the authors of [33] noted that only one detection per image was needed to trigger a spray event, this paper defines a true positive as an image where one or more relevant targets were detected by the CNN. The detection threshold for testing was set at 0.15 .

Using an RTX $2080 \mathrm{Ti}$ GPU on a smart sprayer is not ideal due to the large physical size of desktop computers, and the

${ }^{1} \mathrm{v} 4.52$, Wiener Neustadt, Austria

${ }^{2}$ NVIDIA Corporation, Santa Clara, CA, USA

${ }^{3}$ Intel Corporation, Santa Clara, CA, USA

${ }^{4}$ Canonical Ltd., London, UK 
$650 \mathrm{~W}$ power requirement for a computer containing this GPU. Therefore, mobile processing hardware was tested to determine if it was viable for running multiple CNNs to process images in real-time. An MSI workstation laptop ${ }^{1}$ containing an NVIDIA Quadro RTX 5000 Max-Q 16 GB GPU, an Intel i9-9880H 2.30 $\mathrm{GHz} \mathrm{CPU}$, and $64 \mathrm{~GB}$ of RAM running the Windows 10 Pro $^{2}$ operating system was used for testing. Four USB 2.0 cameras $^{3}$ were plugged into separate USB 3.1 ports on the laptop and the video captured by each camera was processed using a separate instance of Darknet. YOLOv3 and YOLOv3-Tiny were tested at each resolution with 1,2 , and 4 cameras running simultaneously. This test took place indoors instead of in-field conditions, so the CNNs were loaded using weights trained on the $\mathrm{COCO}^{4}$ dataset by [34]. The average processed frames per second (FPS) for each camera reported by Darknet, and the total amount of video RAM (vRAM) needed, as reported by Task Manager, in each test were recorded.

\section{RESULTS AND DISCUSSION}

\section{A. Detection in Static Images}

YOLOv3 and YOLOv3-Tiny were both able to successfully detect if an image contained fescue (Fig. 2). YOLOv3-Tiny produced equal or slightly better results than YOLOv3 for all three metrics at each respective resolution (Table I.). The best F1-score, 0.97, was produced by YOLOv3-Tiny at 1280x736 resolution. Reducing the resolution of YOLOv3-Tiny resulted in a reduction in precision, meaning there were more false

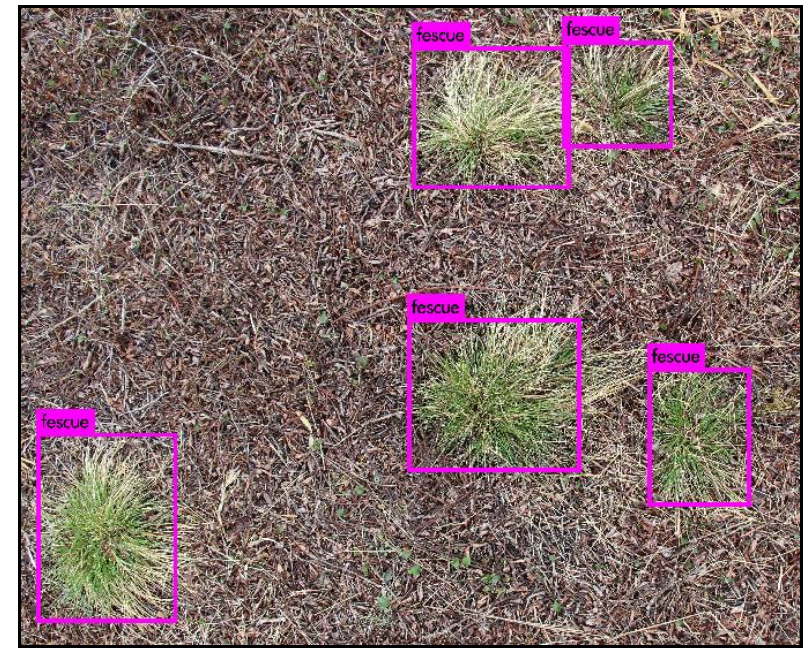

Figure 2. Fescue detections using YOLOv3-Tiny at 1280x736 resolution for an image from the Cattle Market field $\left(45.3674^{\circ} \mathrm{N}\right.$, $63.2124^{\circ} \mathrm{W}$ ) captured on April 24, 2019 [33]. The green and tan bladelike leaves are fescue plants growing in a field with wild blueberry branches leftover from flail mowing the previous autumn. Sheep sorrel is also present in the image.

positive detections, but no change in recall. YOLOv3 saw increases in recall as the resolution was decreased, indicating that higher resolutions resulted in more missed instances of fescue.

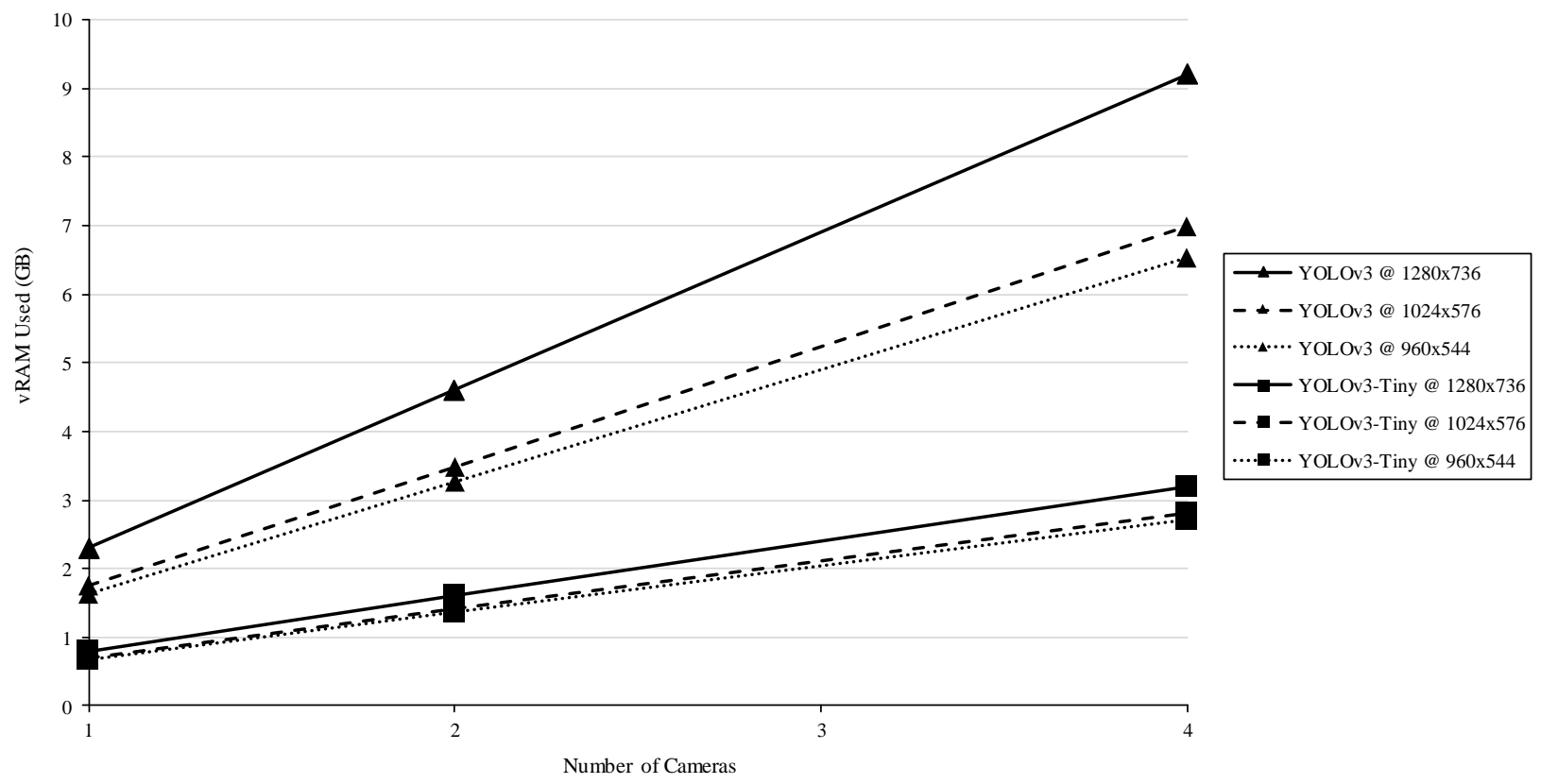

Figure 4. Linear relationships between vRAM usage and number of cameras in simultaneous use for YOLOv3 and YOLOv3-Tiny at various resolutions.

\footnotetext{
${ }^{1}$ WS65 9TM-1410CA, Micro-Star International Co., Ltd., New Taipei, Taiwan

${ }^{2}$ Microsoft Corporation, Redmon, WA, USA

${ }^{3} \mathrm{c} 920$, Logitech International S.A., Lausanne, Switzerland

${ }^{4}$ Common Objects in Context (http://cocodataset.org/)
} 


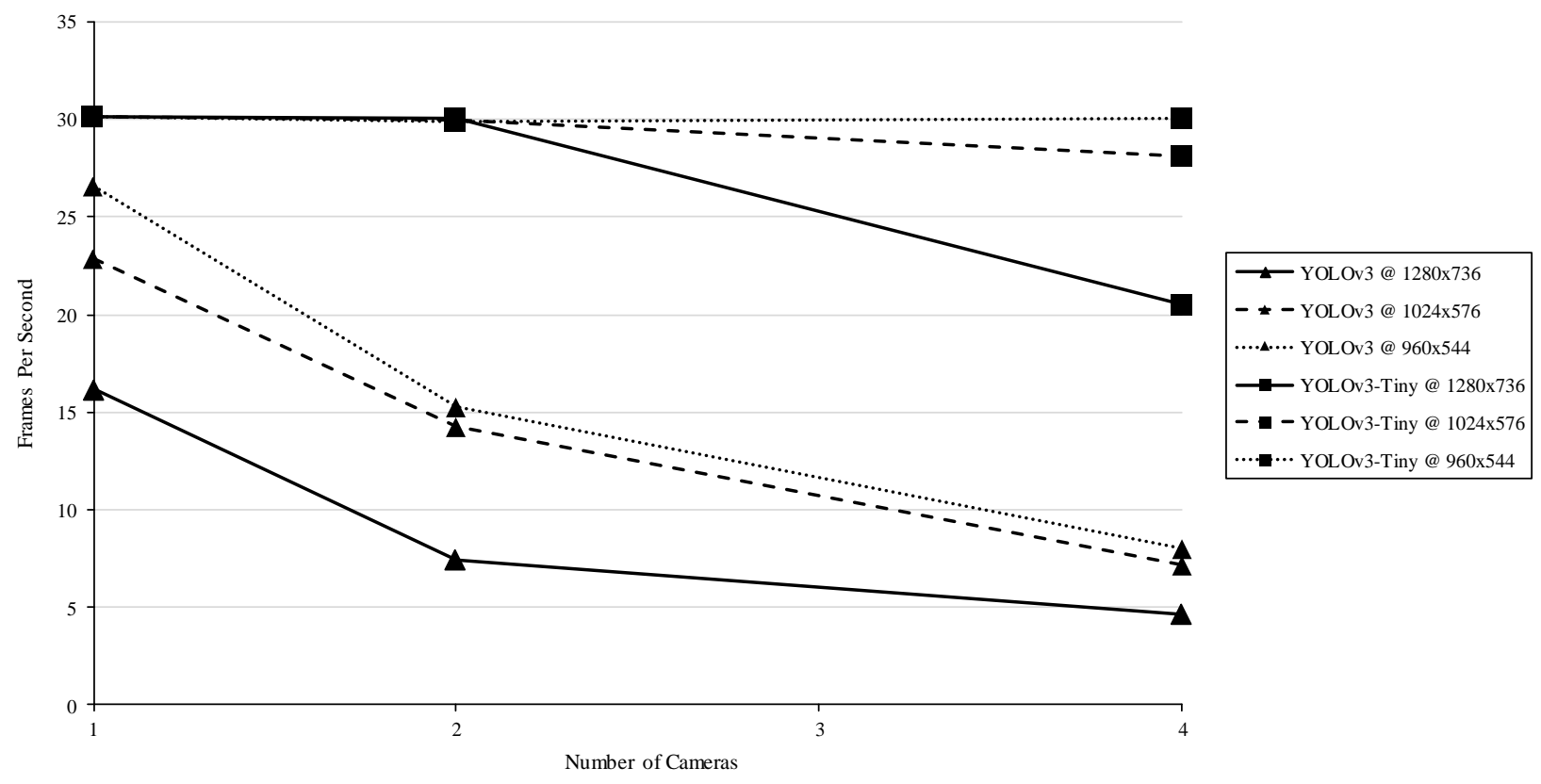

Figure 5. Average processing speed of YOLOv3 and YOLOv3-Tiny for detecting objects at various resolutions and number of cameras.

Detection of sheep sorrel was best achieved using YOLOv3 and 1280x736 resolution, which produced an F1-score of 0.93 (Table II). Sheep sorrel detection with YOLOv3 and 1024x576 and 960x544 resolutions produced precision scores 0.09 and 0.14 higher than the recall. Lowering the detection threshold would increase the recall at the expense of precision and may

TABLE I. RESULTS OF FESCUE DETECTION IN STATIC IMAGES USING YOLOV3 AND YOLOV3-TINY.

\begin{tabular}{ccccc}
\hline Network & $\begin{array}{c}\text { Resolution } \\
\text { (Pixels) }\end{array}$ & Precision & Recall & F1-Score \\
\hline \multirow{3}{*}{ YOLOv3 } & $1280 \times 736$ & 0.97 & 0.93 & 0.95 \\
& $1024 \times 576$ & 0.96 & 0.94 & 0.95 \\
& $960 \times 544$ & 0.96 & 0.95 & 0.95 \\
\hline \multirow{3}{*}{ YOLOv3-Tiny } & $1280 \times 736$ & 0.97 & 0.97 & 0.97 \\
& $1024 \times 576$ & 0.96 & 0.97 & 0.96 \\
& $960 \times 544$ & 0.95 & 0.97 & 0.96 \\
\hline
\end{tabular}

TABLE II. RESULTS OF SHEEP SORREL DETECTION IN STATIC IMAGES USING YOLOV3 AND YOLOV3-TINY.

\begin{tabular}{ccccc}
\hline Network & $\begin{array}{c}\text { Resolution } \\
\text { (Pixels) }\end{array}$ & Precision & Recall & F1-Score \\
\hline \multirow{3}{*}{ YOLOv3 } & $1280 \times 736$ & 0.94 & 0.92 & 0.93 \\
& $1024 \times 576$ & 0.95 & 0.86 & 0.90 \\
& $960 \times 544$ & 0.96 & 0.82 & 0.88 \\
\hline \multirow{3}{*}{ YOLOv3-Tiny } & $1280 \times 736$ & 0.84 & 0.95 & 0.89 \\
& $1024 \times 576$ & 0.83 & 0.95 & 0.88 \\
& $960 \times 544$ & 0.82 & 0.95 & 0.88 \\
\hline
\end{tabular}

result in a higher F1-score. Inversely, sheep sorrel detection with YOLOv3-Tiny produced higher recall than precision scores at all resolutions. Increasing the detection threshold would improve precision at the expense of recall.

\section{B. Processing Performance on a Mobile GPU}

The 16 GB of vRAM included with the RTX 5000 GPU was enough for processing four video streams simultaneously using CNNs. YOLOv3 at $1280 \times 736$ resolution required the most vRAM, 9.2 GB for four video streams, while YOLOv3Tiny at $960 \times 544$ required the least, $2.7 \mathrm{~GB}$ for four video streams (Fig.3). For both CNNs, reducing the resolution resulted in less vRAM usage. This was expected, as there is less information to process in a smaller image. YOLOv3 required more vRAM to process video streams than YOLOv3Tiny at every resolution, indicating that it is a more viable option for processing many video streams on a single GPU. The vRAM requirement was found to scale linearly with the number of cameras. Understanding this relationship will be useful for selecting GPUs for future research. Most currently available GPUs have $8 \mathrm{~GB}$ or less of vRAM, with only the most expensive GPUs containing more. Using YOLOv3-Tiny for processing video streams would be preferable for projects with a modest budget.

There was no discernable change in speed for YOLOv3Tiny at any resolution when processing with one or two cameras but did see reductions when four cameras were in use (Fig. 4). A potential reason for the lack of change is that the CNN was limited by the c920 camera, which captures video at 30 frames per second.

YOLOv3 consistently had framerates of less than 10 FPS when four cameras were in use. The slowest average processing speed was 4.7 FPS at $1280 \times 736$ resolution, while 
the fastest was 8.0 FPS at 960x544 resolution. YOLOv3-Tiny processed video much faster, with framerates between 20.5 FPS and 30.1 FPS. Based on the vRAM usage and processing speed, YOLOv3-Tiny provides a much better option than YOLOv3 for use in a smart sprayer. Considering that YOLOv3-Tiny had the highest F1-score for fescue detection, and only a slightly lower F1-score for sheep sorrel than YOLOv3, it is the better option for use as part of a machine vision system on a smart sprayer. By defining a true positive as an image where one or more relevant targets were detected by the CNN, these object detection CNNs were evaluated as image classification CNNs. Image classifiers only determine the appropriate label for an image, and not the location of objects within an image. It is possible that using an image classifier would result in faster processing speeds and less vRAM usage than the two CNNs evaluated in this paper. Additionally, the latency of the CNNs should be tested to determine the time between an object appearing in front of the camera, and when the CNN detects it. Future work will involve testing image classifiers to compare results and measuring the latency of the system.

\section{CONCLUSIONS}

Previous weed detection systems in wild blueberry smart sprayers have been limited by their inability to discriminate between different plants. Convolutional neural networks can successfully discern between different targets using visual information with minimal direction from the user. YOLOv3 and YOLOv3-Tiny at $1280 \times 736$ resolution produced F1-scores of 0.95 and 0.97 respectively for fescue. For sheep sorrel, the F1-scores were 0.93 for YOLOv3 and 0.89 for YOLOv3-Tiny at the same resolution. Although the accuracy was comparable for both networks, the processing speed and vRAM usage were not. YOLOv3 processed four video streams at 1280x736 resolution at 4.7 FPS using 9.2 GB of vRAM, while YOLOv3Tiny did the same at 20.5 FPS with only 3.2 GB of vRAM. YOLOv3-Tiny provides a faster, lighter, and similarly accurate solution for detecting fescue and sheep sorrel compared to YOLOv3. Image classification networks could potentially provide an even faster and lighter solution for this problem and should be tested in future. Using a convolutional neural network to control spray applications will reduce herbicide use, creating major cost-savings for wild blueberry producers.

\section{ACKNOWLEDGMENTS}

The authors would like to thank Dr. Scott White for helping with field selection for development of the image dataset and the wild blueberry growers for use of their fields during image collection. Also, the authors would like to acknowledge the efforts of the mechanized systems and precision agriculture research teams at Dalhousie's Faculty of Agriculture.

\section{REFERENCES}

[1] D. Robinson, “Acadia Blueberry Price Bulletin,” 2020.

[2] I. V. Hall, L. E. Aalders, N. L. Nickerson, and S. P. Vander Kloet, "The Biological Flora of Canada 1. Vaccinium angustifolium Ait., Sweet Lowbush Blueberry," Can. Field-Naturalist, vol. 93, pp. 415-430, 1979
[3] K. I. N. Jensen and D. E. Yarborough, "An Overview of Weed Management in the Wild Lowbush Blueberry-Past and Present," Small Fruits Rev., vol. 3, no. 3-4, pp. 229-255, 2004.

[4] D. E. Yarborough, "Innovations in weed management in wild blueberry fields in Maine," Acta Hortic., vol. 715, pp. 197-202, 2006.

[5] D. E. Yarborough and P. C. Bhowmik, "Lowbush BlueberryBunchberry Competition," J. Am. Soc. Hortic. Sci., vol. 118, no. 1, pp. 54-62, 1993.

[6] K. V. McCully, M. G. Sampson, and A. K. Watson, "Weed Survey of Nova Scotia Lowbush Blueberry (Vaccinium angustifolium) Fields," Weed Sci., vol. 39, no. 2, pp. 180-185, 1991.

[7] S. N. White, "Final Weed Survey Update and Research Progress on Priority Weed Species in Wild Blueberry," in Wild Blueberry Producers Association of Nova Scotia Annual General Meeting, 2019.

[8] S. N. White and S. K. Kumar, " Potential Role of Sequential Glufosinate and Foramsulfuron Applications for Management of Fescues ( Festuca spp.) in Wild Blueberry ," Weed Technol., vol. 31, no. 1, pp. 100-110, 2017.

[9] K. J. Kennedy, N. S. Boyd, and V. O. Nams, "Hexazinone and Fertilizer Impacts on Sheep Sorrel (Rumex acetosella) in Wild Blueberry," Weed Sci., vol. 58, no. 3, pp. 317-322, 2010.

[10] A. Hughes, S. N. White, N. S. Boyd, P. Hildebrand, and G. Christopher Cutler, "Red sorrel management and potential effect of red sorrel pollen on Botrytis cinerea spore germination and infection of lowbush blueberry (Vaccinium angustifolium Ait.) flowers," Can. J. Plant Sci., vol. 96, no. 4, pp. 590-596, 2016.

[11] T. Esau, Q. U. Zaman, Y. K. Chang, D. Groulx, A. W. Schumann, and A. A. Farooque, "Prototype variable rate sprayer for spotapplication of agrochemicals in wild blueberry," Appl. Eng. Agric., vol. 30, no. 5, pp. 717-725, 2014.

[12] T. Esau et al., "Economic analysis for smart sprayer application in wild blueberry fields," Precis. Agric., vol. 17, no. 6, pp. 753-765, 2016.

[13] T. Esau, Q. Zaman, D. Groulx, A. Farooque, A. Schumann, and Y. Chang, "Machine vision smart sprayer for spot-application of agrochemical in wild blueberry fields," Precis. Agric., vol. 19, no. 4, pp. 770-788, 2018.

[14] T. U. Rehman, Q. U. Zaman, Y. K. Chang, A. W. Schumann, K. W. Corscadden, and T. Esau, "Optimising the parameters influencing performance and weed (goldenrod) identification accuracy of colour co-occurrence matrices," Biosyst. Eng., vol. 170, pp. 85-95, 2018.

[15] T. U. Rehman, Q. U. Zaman, Y. K. Chang, A. W. Schumann, and K. W. Corscadden, "Development and field evaluation of a machine vision based in-season weed detection system for wild blueberry," Comput. Electron. Agric., vol. 162, no. July 2018, pp. 1-13, 2019.

[16] Q. U. Zaman et al., "Development of prototype automated variable rate sprayer for real-time spot-application of agrochemicals in wild blueberry fields," Comput. Electron. Agric., vol. 76, no. 2, pp. 175$182,2011$.

[17] Y. LeCun, Y. Bengio, and G. Hinton, "Deep learning," Nature, vol. 521, no. 7553, pp. 436-444, 2015.

[18] D. Rumelhart, G. Hinton, and R. J. Williams, "Learning representations by back-propagating errors," Nature, vol. 323, 1986.

[19] J. Schmidhuber, "Deep learning in neural networks: An overview," Neural Networks, vol. 61, pp. 85-117, 2015.

[20] A. Kamilaris and F. X. Prenafeta-Boldú, "Deep learning in agriculture: A survey," Comput. Electron. Agric., vol. 147, no. February, pp. 70-90, 2018. 
[21] D. Wu et al., "Lameness detection of dairy cows based on the YOLOv3 deep learning algorithm and a relative step size characteristic vector," Biosyst. Eng., vol. 189, pp. 150-163, 2020.

[22] Q. Yang, D. Xiao, and S. Lin, "Feeding behavior recognition for group-housed pigs with the Faster R-CNN," Comput. Electron. Agric., vol. 155, no. October, pp. 453-460, 2018.

[23] M. M. Santoni, D. I. Sensuse, A. M. Arymurthy, and M. I. Fanany, "Cattle Race Classification Using Gray Level Co-occurrence Matrix Convolutional Neural Networks," Procedia Comput. Sci., vol. 59, no. Iccsci, pp. 493-502, 2015.

[24] J. Amara, B. Bouaziz, and A. Algergawy, "A Deep Learning-based Approach for Banana Leaf Diseases Classification," in BTW 2017, 2017, pp. 79-88.

[25] A. Fuentes, S. Yoon, S. C. Kim, and D. S. Park, "A robust deeplearning-based detector for real-time tomato plant diseases and pests recognition,"Sensors (Switzerland), vol. 17, no. 9, 2017.

[26] A. Venkataramanan, D. K. P. Honakeri, and P. Agarwal, "Plant Disease Detection and Classification Using Deep Neural Networks," Int. J. Comput. Sci. Eng., vol. 11, no. 9, pp. 40-46, 2019.

[27] A. W. Schumann, N. S. Mood, P. D. K. Mungofa, C. MacEachern, Q. U. Zaman, and T. Esau, "Detection of Three Fruit Maturity Stages in Wild Blueberry Fields Using Deep Learning Artificial Neural Networks," in 2019 ASABE Annual International Meeting, 2019, pp. 1-8.

[28] S. M. Sharpe, A. W. Schumann, and N. S. Boyd, "Detection of Carolina geranium (Geranium carolinianum) growing in competition with strawberry using convolutional neural networks," Weed Sci., vol. 67, no. 2, pp. 239-245, 2019.
[29] S. M. Sharpe, A. W. Schumann, J. Yu, and N. S. Boyd, "Vegetation detection and discrimination within vegetable plasticulture rowmiddles using a convolutional neural network," Precis. Agric., no. November, 2019.

[30] J. Yu, S. M. Sharpe, A. W. Schumann, and N. S. Boyd, "Detection of broadleaf weeds growing in turfgrass with convolutional neural networks," Pest Manag. Sci., vol. 75, no. 8, pp. 2211-2218, 2019.

[31] J. Yu, S. M. Sharpe, A. W. Schumann, and N. S. Boyd, "Deep learning for image-based weed detection in turfgrass," Eur. J. Agron., vol. 104, no. November, pp. 78-84, 2019.

[32] J. Yu, A. W. Schumann, Z. Cao, S. M. Sharpe, and N. S. Boyd, "Weed Detection in Perennial Ryegrass With Deep Learning Convolutional Neural Network," Front. Plant Sci., vol. 10, no. October, pp. 1-9, 2019.

[33] P. J. Hennessy, T. J. Esau, Q. U. Zaman, K. W. Corscadden, A. W. Schumann, and A. A. Farooque, "Convolutional Neural Networks for Real-Time Fescue and Sheep Sorrel Identification in Wild Blueberry Fields," in Unpublished Manuscript, 2020, pp. 1-10.

[34] J. Redmon and A. Farhadi, "YOLOv3: An Incremental Improvement," 2018.

[35] J. Redmon, A. Bochkovskiy, and S. Sinigardi, "Darknet: YOLOv3 Neural Network for Object Detection," GitHub Repository, 2020. [Online]. Available: https:/github.com/AlexeyAB/darknet. [Accessed: 20-Jan-2020].

[36] M. Sokolova and G. Lapalme, "A systematic analysis of performance measures for classification tasks," Inf. Process. Manag., vol. 45, no. 4, pp. 427-437, 2009. 